\title{
Genetically engineered mesenchymal stromal cells in cancer gene therapy
}

\author{
Matuskova M, Durinikova E, Altaner C, Kucerova L \\ Cancer Research Institute, Biomedical Research Center, Slovak Academy of Sciences, Bratislava, Slovakia. \\ miroslava.matuskova@savba.sk
}

\begin{abstract}
Based on our experimental data, we aimed to emphasise the perspectives of the use of mesenchymal stromal cells (MSC) in the cancer gene therapy. On the other hand, we would like to point out factors which should be taken into consideration at their clinical use. In this review we define MSC as unique targets for targeted therapy. We proved the efficacy of experimental therapeutic approach utilising enzymatic conversion of non-toxic prodrug into chemotherapeutic by engineered MSC, and we observed significant cytotoxic effect in many preclinical models including metastatic disease. Treatment was enabled by affinity of MSC to tumour tissue and subsequent delivery of therapeutic molecule into the tumour. We also observed decreased efficacy of cell-mediated gene therapy on chemoresistant tumour cells. Moreover MSC can exert a supportive effect on tumour cells as well as to decrease the efficacy of conventional treatment. Besides obvious unique benefits connected to the use of MSC we pointed also to possible risks associated with their clinical application (Ref. 24). Text in PDF www.elis.sk. KEY WORDS: mesenchymal stromal cells, cancer gene therapy, prodrug-converting genes, chemoresistance, tumour microenvironment.
\end{abstract}

\section{Introduction}

Despite advances in diagnostics and therapy, cancer belongs to the major causes of death in western countries. Conventional treatment is accompanied by adverse effects with negative impact on patients' quality of life. Chemoresistance and metastatic dissemination of tumour cells mostly lead to death of oncological patients. It is necessary to search for innovative therapeutic approaches. Cancer gene therapy utilizing enzymatic conversion of non-toxic prodrug into active cytotoxic compound has been used as experimental treatment for many years. Viral vectors, different types on nanocarriers or physical methods have been used for delivery of therapeutic gene into target cells. Preclinical research brought promising results, but clinical studies failed because of inefficient transfer of genetic information into target cells, insufficient infiltration of tumour tissue by vector or low expression of therapeutic gene, respectively. The use of MSC as vehicles for therapeutic genes has enabled to overcome these obstacles as rev in (1).

Cancer Research Institute, Biomedical Research Center, Slovak Academy of Sciences, Dubravska cesta 9, SK-845 05 Bratislava, Slovakia.

Address for correspondence: $\mathrm{M}$. Matuskova, $\mathrm{MD}, \mathrm{PhD}$, Cancer Research Institute, Biomedical Research Center, Slovak Academy of Sciences, Dubravska cesta 9, SK-845 05 Bratislava, Slovakia.

Phone: +421.2.32295138, Fax: +421.2.54774284

Acknowledgement: Slovak Research and Development Agency contracts APVV-0052-12 and APVV-0230-11, RFL and WAC programs funded by the Slovak Cancer Research Foundation, the League against Cancer, VEGA grants No. 2/0171/13 and 2/0130/13.

\section{Mesenchymal stromal cells}

Mesenchymal stromal cells (MSC) represent a population of non-hematopoietic multipotent cells of fibroblastoid morphology. Originally they were identified in bone marrow (2). Till now they have been found almost in all organs (3) located on the abluminal side of blood vessels (4). To the most often used sources of MSC in research as well as in clinical practice belong bone marrow, adipose tissue, dental pulp, umbilical cord or umbilical blood, respectively. The natural function of MSC is to support homeostasis; they contribute to regeneration and wound healing. Based on paracrine intercellular signalling they enter blood stream and home in the side of injury or inflammation (5). The International Society for Cellular Therapy approved a consensus of minimal criteria characterising MSC: ex vivo they are defined as plasticadherent cells when cultured in standard conditions, and they are able to differentiate into osteogenic, adipogenic and chondrogenic lineages. More than $95 \%$ of the cells must express surface markers CD73, CD90 and CD105, and they must lack expression of CD45, CD34, CD14 or CD11b, CD79 $\alpha$ or CD19 a HLA-DR (6). There is an effort to search for new criteria based on advanced methods such as analysis of transcriptome, proteome and secretome which would enable more accurate definition of MSC (7), (8) (1).

\section{Gene therapy mediated by mesenchymal stromal cells}

The finding that MSC possess along other unique features also high affinity to tumour tissue enabled their usage as targeted delivery vehicles for therapeutic molecules, which significantly 


\section{1-223}

contributed to progress in cancer gene therapy. Genetically engineered MSC deliver the therapeutic gene to the tumour based on paracrine signals released by tumour imitating injured tissue (9). These signals are recognised by MSC, they home in tumour and subsequently they become an integral part of tumour stroma. The use of cellular vehicles in gene therapy significantly reduced limitations related to viral or non-viral vectors.

In our laboratory we have been using adipose tissue-derived MSC (AT-MSC) for this purpose. Adipose tissue is really convenient source of MSC; it can be obtained in sufficient quantity from healthy donors at liposuction or plastic surgery. The next advantage is relatively big proportion of AT-MSC within nucleated cells in adipose tissue. The frequency among nucleated cells in bone marrow is $1: 50,000-1: 100,000$, the frequency of MSC in adipose tissue is 500-fold higher (10).

After isolation of AT-MCS by collagenase digestion and plastic adherence we used retroviral vectors for genetic modification. Retroviruses integrate the therapeutic gene into genome of the host cell, and they provide stable expression of transgene and vertical transfer into daughter cells.

More approaches can be used in cancer gene therapy. It is possible to supress the expression of activated oncogenes, restore the expression of tumour-suppressor genes, inhibit tumour angiogenesis and metastatic potential of tumour cells or activate anticancer immunity. Prodrug-converting genes represent important group of therapeutic genes used for gene-directed enzyme prodrug therapy (GDEPT). They encode enzymes which convert non-toxic prodrug into toxic product (11). Our group has been focusing on this type of gene therapy. For genetic modification of AT-MSC we use genes which do not have their equivalent in mammalian cells - 'normal' mammal cells are not able to activate the prodrug. Only engineered cells are capable to convert prodrug to active chemotherapeutic. This approach has been called 'suicide gene therapy' since it causes the death of transgene-expressing cells. For cell-mediated approaches it is not the primary aim to kill the engineered cell (although this phenomenon increases the safety of this therapeutic approach). The cell-mediated approach is based on bystander effect - the aim is to induce apoptosis in neighbouring tumour cells by release of drug converted by therapeutic cells.

The main advantage when compared to conventional chemotherapy is cytotoxic effect localised to the tumour, where prodrug and activating enzyme get together.

We demonstrated that therapeutic systems using fusion gene yeast cytosine deaminase::uracil phosphoribosyl transferase (CD::UPRT) with prodrug 5-fluorocytosine (5-FC) or Herpes simplex virus thymidine kinase (HSVtk) with ganciclovir (GCV) exert high efficacy on many types of tumour cells in vitro as well as on mice models. After systemic administration of therapeutic cells we observed significant inhibition of growth of subcutaneous xenotransplants derived from colorectal cancer (12) or melanoma (13). In this melanoma model we also demonstrated that more than $80 \%$ of treated mice survive for long-term without relapse (14).

We also achieved relevant results on orthotopic glioblastoma multiforme (GBM) model. No effective treatment exists for this aggressive malignant disease, and the prognosis for patients suf- fering from GBM is very unfavourable. We demonstrated that genetically engineered AT-MSC maintain the tumour tropism when they are intracranially administered into distant regions from xenotransplant induced by rat GBM-derived cells C6. The use of osmotic pumps enabling continuous administration of 5-FC and repeated injection of therapeutic cells even prolonged the survival of experimental animals (15). Study which simulated the therapeutic regimen of patients suffering from GBM revealed that CD:UPRT-MSC/5-FC treatment possess curative potential leading to long-term survival of rats, which underwent surgical resection of tumour and subsequent administration of therapeutic cells and continuous administration of 5-FC (16).

We have also focused on treatment of metastatic disease. Ovarian cancer belongs to diseases with unfavourable prognosis because of chemoresistance and abdominal metastases. We achieved long-term survival by CD:UPRT-MSC/5-FC treatment int one third of metastases-bearing mice (17). In a model of lung metastases induced by breast cancer-derived cells we proved that systems CD:UPRT-MSC/5-FC and HSVtk-MSC/GCV act in synergic manner. By combination of two mentioned approaches it is possible to eliminate tumour cells which are resistant to particular systems (18).

On the other hand we demonstrated that intrinsic properties of tumour cells play a key role in efficacy of cell-mediated cancer gene therapy. The ability of gap-junctional intercellular communication (GJIC) is critical especially for HSVtk-MSC/GCV system, because phosphorylated GCV is not able to diffuse across cell membranes, and it passes from therapeutic to target cells via connexin intercellular junctions (19). We observed low efficacy of HSVtk-MSC/GCV approach on tumour cell lines deficient in GJIC. The expression level of enzymes involved in nucleotide metabolism is very important for treatment efficacy as well as expression of $\mathrm{ABC}$ transporters which are responsible for efflux of chemotherapeutics from tumour cells into extracellular space (20).

We demonstrated that various types of tumour cells differ in ability to stimulate the homing of MSC into tumour. Despite promising results in vitro, in mice model we did not achieve significant inhibition of subcutaneous xenografts induced by glioblastomaderived cell line 8-MG-BA. We assume that insufficient infiltration of tumour by therapeutic MSC caused the failure of therapy (19).

A pilot study with genetically-modified neural stem cells expressing E. coli cytosine deaminase for treatment of recurrent high grade gliomas was completed (21), and the second similar study has been active in United States (22). The first European study TREAT-ME1 aimed to assess the safety and efficacy of therapy by autologous MSC isolated from bone marrow transduced with retroviral vector coding HSVtk in combination with GCV in advanced gastrointestinal cancer started in 2015 (23).

A study defining the efficacy of gene therapy mediated by cellular vehicles on chemoresistant populations of tumour cells or cancer stem cells has not been published yet. Chemoresistance of tumour cells is a phenomenon which occurs very often, and it complicates or even disables the cancer treatment. Therefore we focused also on evaluation of efficacy of this therapeutic approach on chemoresistant cells. We cultured tumour cells derived from colorectal carcinoma in consecutively increasing concentration of 
5-FU, and we prepared chemoresistant derivative HT-29/EGFP/ FUR, which proliferate in plasma-relevant concentration of 5-FU. The resistance as well as expression of genes associated with aggressive phenotype increased with numbers of passages, and it negatively influenced the efficacy of cell-mediated cancer gene therapy. We pointed out that it is important to consider previous therapy and possible resistance when defining inclusion criteria for patients entering clinical studies (Durinikova et al., submitted).

In clinical use of MSC it is important to take into consideration that MSC (or cells derived from them) contribute to tumour microenvironment, where they influence the properties of tumour cells. As mentioned above, MSC expressing prodrug-converting genes are affected by toxic metabolites produced by inserted enzyme and despite their relative high resistance they undergo apoptosis in time. This fact enables GDEPT mediated by MSC to be declared safe.

Non-engineered MSC are used in regenerative medicine, plastic surgery, for treatment of autoimmune diseases and GVHD. It was proved that MSC as integral part of tumour microenvironment can support proliferation, invasiveness, and they can decrease the sensitivity to chemotherapy. We demonstrated that MSC cultured in the presence of cisplatin differ from cells cultured without chemotherapeutic. Alterations in secretion and phosphorylation profile of MSC subsequently negatively influenced the response of breast cancer-derived cells to chemotherapy, and supported expression of cancer stem cells' markers (24). It is necessary to understand the tumour cells-MSC interplay to maximise the safety of cell therapies.

\section{Learning points}

The therapeutic potential of MSC is incontestable. Several clinical studies using MSC or progenitor cells for treatment of various conditions have been conducted. MSC are used in the treatment of autoimmune disorders and graft-versus-host disease (GVHD), as well as in regenerative medicine and plastic surgery. These unique cells enabled significant progress in cancer gene therapy. On the other hand it is necessary to think of the fact that MSC can exert supportive effect on tumour cells, and they can decrease the efficacy of conventional cancer treatment.

\section{References}

1. Durinikova E, Kucerova L, Matuskova M. Mesenchymal stromal cells retrovirally transduced with prodrug-converting genes are suitable vehicles for cancer gene therapy. Acta Virol 2014; 58 (1): 1-13.

2. Friedenstein AJ, Chailakhjan RK, Lalykina KS. The development of fibroblast colonies in monolayer cultures of guinea-pig bone marrow and spleen cells. Cell Tissue Kinet 1970; 3 (4): 393-403.

3. da Silva Meirelles L, Chagastelles PC, Nardi NB. Mesenchymal stem cells reside in virtually all post-natal organs and tissues. J Cell Sci 2006; 119 (11): 2204-2213.

4. Crisan M, Yap S, Casteilla L et al. A perivascular origin for mesenchymal stem cells in multiple human organs. Cell Stem Cell 2008; 3 (3): 301-313.

5. Strioga M, Viswanathan S, Darinskas A, Slaby O, Michalek J. Same or not the same? Comparison of adipose tissue-derived versus bone marrowderived mesenchymal stem and stromal cells. Stem Cells Dev 2012; 21 (14): 2724-2752.
6. Dominici M, Le Blanc K, Mueller I et al. Minimal criteria for defining multipotent mesenchymal stromal cells. The International Society for Cellular Therapy position statement. Cytotherapy 2006; 8 (4): 315-317.

7. Keating A. Mesenchymal stromal cells: new directions. Cell Stem Cell 2012; 10 (6): 709-716.

8. Ranganath SH, Levy O, Inamdar MS, Karp JM. Harnessing the mesenchymal stem cell secretome for the treatment of cardiovascular disease. Cell Stem Cell 2012; 10 (3): 244-258.

9. Dvorak HF. Tumors: wounds that do not heal. Similarities between tumor stroma generation and wound healing. N Engl J Med 1986; 315 (26): 1650-1659.

10. Fraser JK, Wulur I, Alfonso Z, Hedrick MH. Fat tissue: an underappreciated source of stem cells for biotechnology. Trends Biotechnol 2006; 24 (4): 150-154.

11. Portsmouth D, Hlavaty J, Renner M. Suicide genes for cancer therapy. Mol Aspects Med 2007; 28 (1): 4-41.

12. Kucerova L, Altanerova V, Matuskova M, Tyciakova S, Altaner C. Adipose tissue-derived human mesenchymal stem cells mediated prodrug cancer gene therapy. Cancer Res 2007; 67 (13): 6304-6313.

13. Kucerova L, Matuskova M, Pastorakova A et al. Cytosine deaminase expressing human mesenchymal stem cells mediated tumour regression in melanoma bearing mice. J Gene Med 2008; 10 (10): 1071-1082.

14. Kucerova L, Skolekova S, Demkova L, Bohovic R, Matuskova M. Long-term efficiency of mesenchymal stromal cell-mediated CD-MSC/5FC therapy in human melanoma xenograft model. Gene Ther 2014; 21 (10): 874-887.

15. Altanerova V, Cihova M, Babic $\mathbf{M}$ et al. Human adipose tissue-derived mesenchymal stem cells expressing yeast cytosinedeaminase::uracil phosphoribosyltransferase inhibit intracerebral rat glioblastoma. Int J Cancer 2012; 130 (10): 2455-2463.

16. Altaner C, Altanerova V, Cihova $\mathrm{M}$ et al. Complete regression of glioblastoma by mesenchymal stem cells mediated prodrug gene therapy simulating clinical therapeutic scenario. Int J Cancer 2014; 134 (6): 1458-1465.

17. Toro L, Bohovic R, Matuskova M, Smolkova B, Kucerova L. Metastatic Ovarian Cancer Can Be Efficiently Treated by Genetically Modified Mesenchymal Stromal Cells. Stem Cells Dev 2016.

18. Matuskova M, Kozovska Z, Toro L et al. Combined enzyme/prodrug treatment by genetically engineered AT-MSC exerts synergy and inhibits growth of MDA-MB-231 induced lung metastases. J Exp Clin Cancer Res 2015; 34: 33 .

19. Matuskova M, Hlubinova K, Pastorakova A et al. HSV-tk expressing mesenchymal stem cells exert bystander effect on human glioblastoma cells. Cancer Lett 2010; 290 (1): 58-67.

20. Matuskova M, Baranovicova L, Kozovska $Z$ et al. Intrinsic properties of tumour cells have a key impact on the bystander effect mediated by genetically engineered mesenchymal stromal cells. J Gene Med 2012; 14 (12): 776-787.

21. https://clinicaltrials.gov/ct2/show/NCT01172964?term=cytosine+de aminase \&rank=1.

22. https://clinicaltrials.gov/ct2/show/NCT02015819?term=cytosine+de aminase\&rank $=2$.

23. Niess H, von Einem JC, Thomas MN et al. Treatment of advanced gastrointestinal tumors with genetically modified autologous mesenchymal stromal cells (TREAT-ME1): study protocol of a phase I/II clinical trial. BMC Cancer 2015; 15: 237.

24. Skolekova S, Matuskova M, Bohac M et al. Cisplatin-induced mesenchymal stromal cells-mediated mechanism contributing to decreased antitumor effect in breast cancer cells. Cell Commun Signal 2016; 14: 4 .

Received December 21, 2017. Accepted January 9, 2018. 